\title{
Speed optimizations for liner networks with business constraints
}

Reinhardt, Line; Pisinger, David; Sigurd, Mikkel; Ahmt, Jonas

Published in:

European Journal of Operational Research

DOI:

10.1016/j.ejor.2020.02.043

Publication date:

2020

Document Version

Peer reviewed version

Citation for published version (APA):

Reinhardt, L., Pisinger, D., Sigurd, M., \& Ahmt, J. (2020). Speed optimizations for liner networks with business constraints. European Journal of Operational Research, 285(3), 1127-1140.

https://doi.org/10.1016/j.ejor.2020.02.043

\section{General rights}

Copyright and moral rights for the publications made accessible in the public portal are retained by the authors and/or other copyright owners and it is a condition of accessing publications that users recognise and abide by the legal requirements associated with these rights.

- Users may download and print one copy of any publication from the public portal for the purpose of private study or research.

- You may not further distribute the material or use it for any profit-making activity or commercial gain.

- You may freely distribute the URL identifying the publication in the public portal.

\section{Take down policy}

If you believe that this document breaches copyright please contact rucforsk@kb.dk providing details, and we will remove access to the work immediately and investigate your claim. 


\title{
Speed optimizations for liner networks with business constraints
}

\author{
Corresponding author: Line Blander Reinhardt \\ Department of People and Technology, Roskilde University, Universitetsvej 1, 4000 \\ Roskilde, Denmark \\ email: liner@ruc.dk
}

\section{David Pisinger}

DTU Management Engineering, Technical University of Denmark, Produktionstorvet 424, 2800 Kgs. Lyngby, Denmark

Mikkel Muhldorff Sigurd

Maersk Line, Esplanaden 50, 1098 Copenhagen K., Denmark

Jonas Ahmt

Maersk Line, Esplanaden 50, 1098 Copenhagen K., Denmark 


\title{
Speed optimizations for liner networks with business constraints
}

\author{
Line Blander Reinhardt ${ }^{\mathrm{a}, *}$, David Pisinger ${ }^{\mathrm{b}}$, Mikkel M. Sigurd ${ }^{\mathrm{c}}$, Jonas Ahmt ${ }^{\mathrm{c}}$ \\ ${ }^{a}$ Department of People and Technology, Roskilde University, Universitetsvej 1, 4000 Roskilde, Denmark \\ ${ }^{b}$ DTU Management Engineering, Technical University of Denmark, Produktionstorvet 424, 2800 Kgs. Lyngby, Denmark \\ ${ }^{c}$ Maersk Line, Esplanaden 50, 1098 Copenhagen K., Denmark
}

\begin{abstract}
In 2018 the International Maritime Organization (IMO) agreed to cut the shipping sector's overall CO2 output by $50 \%$ by 2050 . One of the key methods in reaching this goal is to improve operations to limit fuel consumption. However, it is difficult to optimize speed for a complete liner shipping network as routes interact with each other, and several business constraints must be respected. This paper presents a unified model for speed optimization of a liner shipping network, satisfying numerous real-life business constraints. The speed optimization is in this research achieved by rescheduling the port call times of a network, thus, the network is not changed. The business constraints are among others related to transit times, port work shifts and emission control areas. Other restrictions are fixed times for canal crossing, speed restrictions in the piracy areas and desire for robust solutions. Vessel sharing agreements and other collaboration between companies must also be included. The modeling of the different restrictions is described in detail and tested on real-life data. The scientific contribution of this paper is threefold: We present a unified model for speed optimization together with numerous business constraints. We present a general framework for handling routes with different frequencies. Moreover, we present a bi-objective model for balancing robustness of schedules against fuel consumption. The tests show that the real-life requirements can be handled by mixed integer programming and that the model finds significant reductions of bunker consumption and cost for large-scale real-life instances.
\end{abstract}

Keywords: OR in maritime industry; speed optimization, CO2, robustness, bi-objective

\section{Introduction}

The International Maritime Organization (IMO) member state delegates have agreed on a target to cut the shipping sector's overall CO2 output by $50 \%$ in 2050 . Despite the ambitious goals, several critics point out the lack of action plan, and urges the industry to transform these goals into concrete, urgent steps. IMO proposes a list of tools that can be used to reach the goal, where one of the most concrete steps is speed reduction and speed optimization.

In this paper we study speed optimization of a complete liner shipping network, while ensuring that all transported goods arrive within the same time frame as previously, or with a negligible delay. In order to make the approach applicable in practice, we consider a huge list of business constraints, that must be addressed before the speed optimization model can be widely adapted by the shipping industry.

We also study robustness of the final schedule, making it possible to make a quantitatively supported trade-off between fuel consumption and schedule robustness. Following the ideas of Weide et al. [36] we define a schedule to be operationally robust if it contains few critical connections. The model is formulated as a bi-objective problem, and we demonstrate that it is possible to find an $\epsilon$-efficient set of optimal solutions.

Most liner shipping companies operate on a published weekly schedule. The vessels continuously sail a specific round trip called a service. Such a service consists of a set of direct connections between two ports

*Corresponding Author, Email liner@ruc.dk, Tel.: +45 41403559 
also called legs, and the service is sailed by a number of vessels of the same type to ensure the same capacity every week. The number of vessels deployed to a service correspond to the number of weeks required for completing the round trip of the service. These services are often designed according to strategic decisions, such as offering a service to take market shares from competitors or offering services in an upcoming market in order to be first movers. As a result of these outside factors the companies prefer to manually design the network. Therefore, we here focus solely on the scheduling and speed optimization of an already designed and operating network. Thus, network design is not part of this research. Moreover, cargo flow has many considerations such as time available in port, efficiency in port, cabotage rules, port accident rate, sanctions and other measures which requires a considerable amount of additional data which currently is difficult to acquire in most companies. In order to ensure that the here presented results are easy to implement for companies, the cargo flow is considered fixed. The speed optimization occurs by simply moving the port call times. Even though vessels do not always arrive on time, the berths at most major container ports must be booked as a fixed weekly slot. Thus, the uncertainty of actual arrival times is not considered, however, note that the more time you have to complete a given journey the higher punctuality can be achieved.

Since the bunker consumption per day grows cubically with the speed, it is possible to save bunker by avoiding large speed fluctuations on the round trip of a service. In this paper the duration of a round trip is fixed. This ensures that the round trip can be served by an unchanged number of vessels, making it easier for shipping companies to implement the strategy. However, a company may solve the model using additional vessels on a service in order to find the possible savings by slowing down the service with the respective number of weeks. Considering only a single round trip without other constraints, the minimal bunker consumption can be achieved by sailing with the average speed on all legs. In practice the company must satisfy a given service level, taking into account not only the cost but also the transport duration of shipping a container. A duration limit not only imposes a lower limit on the speed for a single service, but may affect the speed of all services involved in a transportation chain. In particular one must take into account that when a transshipment to another service is missed, the container will have to wait up to a week for the arrival of the next vessel on the service transshipped to. We will denote the time limit on a transport from location $A$ to location $B$ as transit time limit. This time limit includes the time required for transshipment in ports. Moreover, changing the port call time of a vessel is often a lengthy process involving a significant amount of administrative work, thus a penalty for changing the port call time is introduced in the objective function representing incurred costs.

The basic problem of speed optimization has already been dealt with in Reinhardt et al. [28]. Both in the present paper and in the problem solved in [28] the speed optimization is applied to a given network with given cargo routes and transit time requirements. The present paper extends the model presented in [28] to handle a large number of real-life business constraints that are necessary to take into account when optimizing the speed of rotations. These constraints include among others, work shift times in ports, passage times at canals, emission control areas, piracy areas, and schedule robustness.

Port work shift times and passage times at canals, such as Suez and Panama, impose constraints on the arrival times at ports and along the route. In the emission control areas (ECA) the use of the cheaper high sulphur fuel is forbidden; thus, introducing higher bunker costs. In the piracy areas sailing at fast speeds is necessary, in order to reduce the risk of pirate encounters.

Due to the growing collaboration between liner shipping companies, it is important to also include connections operated by other operators, which may be used for part of the journey. Here it is discussed how to include collaboration especially in the complicated case of feeder liners operating with a different frequency.

All of the mentioned real-life constraints are modeled in this paper, and tested on a real-life case of a major liner shipping company. Test results are presented for the different situations and the increased complexity is discussed.

The scientific contribution of this paper is threefold:

- We present a unified model for speed optimization taking numerous business constraints into account. The detailed modeling allows for shipping companies to easily adapt the model into their scheduling work.

- We present a general framework for handling routes with different frequencies. The framework can 
be used in other transport planning problems where services operate at different frequencies.

- We present a bi-objective model for balancing robustness of the schedule against fuel consumption. We define a schedule to be operationally robust [36] if it evades critical legs. A leg is critical if the slack time is below a given threshold value. Using the epsilon-method, we can find the $\epsilon$-efficient Pareto optimal frontier. Until now, robustness has been handled by manually adding buffers, without having a global overview of how decisions affect the whole shipping network and in particular fuel consumption.

The remainder of the paper is structured as follows: In Section 2 the most recent research in the area is discussed. In Section 3.1 the bunker consumption model used in this paper is shortly described. Next, Section 3 presents the main model for speed optimization of a liner network. The base model for the problem is presented in Section 3.2. Sections 3.3 to 3.10 contains modeling extensions and discussions related the topics:

- Canal convoy times (Section 3.3)

- Work shift regulations (Section 3.4)

- Emission control areas (Section 3.5)

- Piracy precautions (Section 3.6)

- Penalty and bonus for respectively late or early arrival of containers. (Section 3.7)

- Integrating feeder services with different frequencies (Section 3.8)

- Vessel sharing agreements (Section 3.9)

- Operational robustness of schedules (Section 3.10)

In Section 4 we present real-life test cases and results for the above constraints under different model generalizations. The paper is concluded in Section 5 with a discussion of the presented results and how they can contribute to reach the CO2-emission goals defined by IMO.

\section{Literature review}

In this section relevant literature in liner shipping is presented. For a detailed literature review of cargo shipping optimization problems in general, we refer to the papers by Christiansen et al. [8, 9, 7]. For an overview of optimization problems in liner shipping see [4].

Some of the first papers in liner shipping focused on network design as presented in Rana and Vickson [25], Agarwal and Ergun [1], Reinhardt and Kallehauge [26], Alvarez [3], Reinhardt and Pisinger [27] and Brouer et al. [5]. However, as mention by Brouer et al. [5], each paper studied a different version of the network design problem. Moreover, network design in practice is often partly based on strategic decisions which are difficult to quantify in a model. Therefore some recent papers have focused on operational problems in liner shipping companies. One example of such a problem aims at reducing bunker consumption and hence $\mathrm{CO} 2$ emission by changing the port call times, and thereby the speed on each leg. This problem is denoted berth time rescheduling or speed optimization.

A recent review of liner shipping with transit times and berth time scheduling is presented in Reinhardt et al. [28]. The paper gives a detailed overview of recent publications, categorizing them according to the constraints included (e.g. transshipment, round trips, transit time), bunker consumption function, problem type, and solution method [28, Table 1].

One of the first models developed for speed optimizing liner services considering transit times from a set of schedules was proposed by Wang and Meng [32]. The problem studied by these authors considers transit times which include transshipment times for the cargo and probabilistic arrival times. However, the business constraints presented in this paper are not considered in [32] and therefore the work cannot be used in an operating liner shipping company. Wang and Meng [32] solve medium size problems using 
a heuristic and include a function representing the reduction in demand with increasing transit time. In a later paper, Wang and Meng [34] investigate different bunker consumption curves to find the best fitting to real-life bunker consumption. Wang [31] speed optimize a single round trip with respect to transit times without considering transshipment. Karsten et al. [20] solve the container routing problem with transit times and show that it can be used in the network design problem with speed optimization. The problem solved in [20] considers routing the container orders optimally on a network subject to transit time requirements. The research presented in [20] is on a more strategic level as it does not include a schedule for the network and thus the arrival and departure times at ports are unknown. Therefore it is uncertain if a connection is missed but a standard time used on transshipment is assumed to be a fixed value of 24 hours and it is not known if this is actually feasible when implemented. In a recent paper by Karsten et al. [19] a network design problem is solved which simultaneously optimizes the container routing with transit time constraints using Benders' decomposition. In this paper a rough estimate for the time used for transshipment is however still used. A similar problem is solved by Guericke and Tierney [16] in which the duration of the port stays are adjusted depending on the amount of containers unloaded and loaded. None of the mentioned papers consider canal traversal times and work shift times. Although the time used at a port is included in some of the mentioned papers the actual operations at the port are not handled, as this is considered a container terminal operation problem (see Iris et al. [18]). Ozcan et al. [21] minimizes the tardiness of cargo by synchronizing transshipments. In [21] the cargo is allocated on routes and the port calls synchronized for cargo transshipment, however, the bunker consumption is not considered.

A recent paper by Wang et al. [35] considers the container flow on a provided network schedule while considering transit times. They propose to model different service frequencies by using the smallest common multiple of the service frequency. This method is also used in the present paper. Different frequencies do, however, not occur in the instances tested by Wang et al. [35] and rescheduling of port calls is not handled as the network and schedule are kept fixed. This paper includes the rescheduling of services, assuming a fixed container assignment.

Bunker consumption in relation to emission control areas is a recent but nonetheless quite active research area. In an early survey by Psaraftis and Kontovas [24] it is noted that research shows that the increased cost of bunker satisfying the emission requirements in emission control areas would (if cost is minimized) results in lower speeds inside the areas and higher speeds outside, thus increasing the overall emission when compared to the overall emission at average speed. A later paper by Fagerholt et al. [12] studies the optimization of speeds with respect to cost and sailing paths for direct connections between two ports for a predefined set of sailing paths. Also, recently, Fagerholt and Psaraftis in [13] optimized profit per time unit to analytically find the optimal refraction point for an optimized sailing path. Wang and Xu [30] studied speed optimization under different emission taxation strategies and the resulting cost and overall emissions. Recently Passchyn et al. [22] used speed optimization to schedule locks so that the combined bunker consumption and thus also emission were reduced within the system containing the locks. Finally Dithmer et al. [10] studied optimizing the bunker cost versus the external cost of the emissions in a single service network design. Wang and Meng [33] consider robust schedules modeled as a mixed-integer nonlinear stochastic programming model. However, Such models are rarely used in real-life applications as they are complex and requires accurate information on the uncertainties. Therefore the simple robustness measure of adding buffer is often applied [14]. The objective is to minimize bunker consumption complying to emission regulations.

To the best of our knowledge this is the first paper on speed optimization including port work shifts and piracy areas. Moreover, the paper presents a unified model for adjusting the robustness of a schedule, and proposes a bi-objective formulation where robustness can be weighed against fuel consumption. Finally, this paper contributes to the literature by calculating transit times using services with different frequencies, and by including a large number of different real-life requirements.

\section{Modeling business constraints and robustness}

In this section the base model and the different business constraints are described. This also includes the modeling of services with different frequencies and a bi-objective model for robustness. 




Figure 1: A bunker curve approximated with 15 secants. The sailing time used on a given leg is presented in hours on the $\mathrm{x}$-axis. The $\mathrm{y}$-axis is the cost of the bunker used to sail the leg.

\subsection{Bunker consumption}

In this subsection we present an LP formulation of bunker consumption as function of speed. Both Stopford [29] and Alderton [2] argue that the bunker consumption per day is a cubic function of speed. Wang and Meng [34] consider several different power function approximations including the cubic function. In the present paper the bunker curves are provided by a company based on historic data of fuel consumption. Without loss of generality it is here assumed that the bunker consumption is convex in the time interval for which the function is defined. Measuring errors may cause historic bunker consumption measurements to have lumps, but this can be resolved by finding the convex hull using the well known Graham scan algorithm [15]. A secant approximation of the bunker function is used to linearize the bunker consumption as a function of time. Note that using a set of secants to approximate the function can be applied to any convex function. Thus, individual bunker consumption curves can be used for each vessel type.

Let a leg be a specific journey from a port $i$ to a port $j$ and let $C_{l}$ be the consumption of sailing a leg $l$ using the time $\tau_{l}$ to complete the sailing. Then $C_{l}$ must satisfy each of the inequalities

$$
C_{l} \geq \phi_{l}^{p} \tau_{l}+\omega_{l}^{p}, \quad p \in P_{l}
$$

where $\phi_{l}^{p}$ is the gradient of secant $p$ on leg $l$ and $\omega_{l}^{p}$ is the constant of the secant $p$ on leg $l$. Figure 1 shows a convex bunker curve on a leg approximated by using secants of the form described in equation (1). We use bunker curves provided by the liner shipping company based on historic data combined with knowledge about the load, vessels and currents.

\subsection{Base model for speed optimization}

This section describes the base model for minimizing bunker consumption of liner services by berth time rescheduling based on the model proposed in [28]. The information relevant for the present paper is presented here, further details may be found in [28]. In the following section the extensions to the model are described.

An overview of the notation can be seen in Table 1. Moreover we use the notation $\left(l^{\prime}, l\right) \in L$ to denote that $l$ is the succeeding leg after $l$ on a service. The model now becomes: 


\begin{tabular}{|c|c|c|}
\hline type & symbol & description \\
\hline sets & $\begin{array}{l}R \\
L \\
Q \\
O \\
P_{l}\end{array}$ & $\begin{array}{l}\text { set of services } \\
\text { set of legs where } l \in L_{r} \text { is a leg on service } r \in R \\
\text { set of demands defined by a route (as set of legs } l \in L \text { ) between two ports } A \text { to } B \\
\text { set of connections between two services } r \text { and } s \text { in } R \text {, transshipping from leg } l \in L_{r} \text { to leg } \hat{l} \in L_{s} \\
\text { set of secants used for approximating the bunker curve on leg } l \in L\end{array}$ \\
\hline parameters & \begin{tabular}{|l}
$\hat{P}_{l}$ \\
$F_{w}$ \\
$F_{d}$ \\
$S_{r}$ \\
$P_{l}^{s}$ \\
$A_{l}$ \\
$T_{l}$ \\
$D_{q}$ \\
$H_{c}$ \\
$K_{c}^{w}$ \\
$\phi_{l}^{p}$ \\
$\omega_{l}^{p}$ \\
$\alpha_{l}$ \\
\end{tabular} & $\begin{array}{l}\text { penalty for shifting a berth time at leg } l \in L \\
\text { the frequency of all vessels, measured in hours. Here } F_{w} \text { is always } 168 \text { hours (weekly) } \\
\text { the frequency of all canals, measured in hours. Here } F_{d} \text { is always } 24 \text { hours (daily) } \\
\text { number of } F_{w} \text { (here weeks) used for the round trip of a service } r \\
\text { the port stay at the end port of leg } l \in L \\
\text { current scheduled time for the port call at leg } l \in L \\
\text { minimum time required for sailing leg } l \in L \text { (at maximum speed) } \\
\text { transit time requirement of demand } q \in Q \\
\text { minimum time required for connection } c \in O \\
\text { equivalent to }\left\lfloor H_{c} / F_{w}\right\rfloor \text { for connection } c \in O \text {. Thus the number of whole } F_{w} \text { (here weeks) in } H_{c} \\
\text { gradient of secant } p \in P \text { of leg } l \in L \\
\text { the value of secant } p \in P \text { of leg } l \in L \text { at duration time equal to zero } \\
\text { weighting factor for robustness } \rho_{l}\end{array}$ \\
\hline variables & $\begin{array}{l}f_{l} \\
t_{l}^{d} \\
t_{l}^{a} \\
C_{l} \\
w_{l}^{d} \\
a_{c} \\
m_{l} \\
x_{c} \\
\rho_{l} \\
\mu\end{array}$ & $\begin{array}{l}\text { (binary) indicates if leg } l \text { is a start leg of a service } \\
\text { (continuous) departure time of leg } l \in L \text { at its end port } \\
\text { (continuous) arrival time of leg } l \in L \text { at its end port } \\
\text { (continuous) cost of sailing leg } l \in L \\
\text { (integer) number of } F_{w} \text { (here weeks) from time zero to } t_{l}^{d} \text { for } l \in L,\left(w_{l}^{d} F_{w} \leq t_{l}^{d}\right) \\
\text { (binary) is one if } t_{c}^{a}+F_{w}\left(K_{c}^{w}-w_{h}^{d}\right)-H_{c}<0 \text { for } c \in O \text { (arriving leg in the previous week) } \\
\text { (binary) is one if the port call time has been changed of leg } l \in L \\
\text { (binary) is one if a week of transshipment needed to ensure transshipment at the connection } c \\
\text { (continuous) robustness (i.e. slack) at leg } l \\
\text { (continuous) robustness (i.e. slack) at all legs }\end{array}$ \\
\hline
\end{tabular}

Table 1: Overview of notation used in the model

$$
\min \sum_{r \in R} \sum_{l \in L_{r}}\left(C_{l}+\hat{P}_{l} m_{l}\right)
$$

$$
\begin{array}{lr}
\sum_{l \in L_{r}} f_{l}=1, & r \in R \quad(3) \\
t_{l}^{a}-t_{l^{\prime}}^{d}+F_{w} S_{r} f_{l} \geq T_{l}, & r \in R,\left(l^{\prime}, l\right) \in L_{r} \quad(4) \\
A_{l}-\left(t_{l}^{d}-F_{w}\left(w_{l}^{d}\right)+F_{w} m_{l}\right) \geq 0, & r \in R, l \in L_{r} \quad(5) \\
t_{l}^{d}-F_{w} w_{l}^{d}-A_{l}+F_{w} m_{l} \geq 0, & r \in R, \quad l \in L_{r} \quad(6) \\
\phi_{l}^{p}\left(t_{l}^{a}-t_{l^{\prime}}^{d}+F_{w} S_{r} f_{l}\right)+\omega_{l}^{p} \leq C_{l}, & r \in R,\left(l^{\prime}, l\right) \in L_{r}, p \in P_{l} \quad(7) \\
0 \leq t_{l}^{d}-F_{w} w_{l}^{d}<F_{w}, & r \in R, l \in L_{r} \quad(8) \\
t_{h}^{a}-H_{c}+F_{w}\left(K_{c}^{w}-w_{h}^{d}+a_{c}\right) \geq 0, & c=(l, h) \in O \quad(9) \\
t_{h}^{a}-H_{c}+F_{w}\left(K_{c}^{w}-w_{h}^{d}+a_{c}\right)<168, & c=(l, h) \in O(10) \\
t_{h}^{a}-t_{l}^{d}-H_{c}+F_{w}\left(w_{l}^{d}+K_{c}^{w}-w_{h}^{d}+x_{c}+a_{c}\right) \geq 0, & c=(l, h) \in O(11) \\
\quad \sum\left(t_{h}^{a}+F_{w}\left(K_{c}^{w}-w_{h}^{d}\right)-t_{l}^{d}+F_{w}\left(w_{l}^{d}+x_{c}\right)\right)+\sum_{c=(l, h) \in O_{q}} & \left(t_{l}^{d}-t_{l^{\prime}}^{d}+F_{w} S_{r} f_{l}\right)-P_{q}^{s} \leq D_{q}, \\
w_{l}^{d} \in\left\{0, \ldots, S_{r}-1\right\}, & q \in Q(12) \\
t_{l}^{a}, t_{l}^{d} \geq 0, C_{l} \geq 0, & r \in R, l \in L_{r}(13) \\
f_{l}, m_{l}, a_{c} \in\{0,1\}, & r \in R, l \in L_{r}(14) \\
x_{c} \in\{0,1\}, & r \in R, l \in L_{r}(15)
\end{array}
$$$$
r \in R, l \in L_{r}(13)
$$ 
The objective (2) minimizes the sum of bunker cost and the penalties for moving port call times. The parameter $\hat{P}$ is the penalty for moving the port call time at the end of leg $l \in L$, and the binary variable $m_{l}$ is one iff the port call time has been moved. The parameter $C_{l}$ is the bunker cost on leg $l$.

The first constraints in the model (3) ensure that for each service exactly one leg is chosen as the first leg of the service. Constraints (4) ensure that the legs are not traversed at a faster speed than the maximum speed of the vessel. Constraints (5) and (6) ensure that a penalty is applied if the port time is changed from the original scheduled (weekly) port call time $A_{l}$ for leg $l \in L$. For every leg the consumption is restricted by a set of linear functions represented by constraints (7). In constraints (7) the variable $\phi_{l}^{p}$ is the slope of secant $p_{l}$ on leg $l \in L$ and $\omega_{l}^{p}$ is the intersection of the secant. Constraints (8) define the value of $w_{l}^{d}$. In (9) and (10) it is ensured that when subtracting the connection time part which is less than a whole week the arrival time of leg $h$ of connection $c=(l, h)$ is positive. If the value is negative, the binary variable $a_{c}$ is used to add a week, such that the adjusted arrival time is a positive value within a week. This will ensure that all calculations are kept within the same week. Constraints (11) ensure that the containers transshipped from $l$ on service $r$ to $h$ on service $s$ waits at the terminal for the next vessel arriving after the minimum required connection time $H_{c}$. The transit time is ensured to be below the requirement $D_{q}$ for demand $q \in Q$ with constraints (12). Constraints (13) to (16) define the variable domains. The reader is referred to [28] for a more thorough explanation of the model presented in this section and note that this model simply serves as a basis for the research presented in the remainder of this paper.

\subsection{Canal passing}

Most liner vessels going from Asia to Europe pass through the Suez Canal. The passage through a canal takes place in a convoy. The convoy will for each direction be scheduled at fixed times, usually once or twice a day. Therefore, it is necessary for the vessels to arrive at the canal entrance at a specific time scheduled by the operators of the canal. The conditions described here are present at congested pathways such as the Panama canal, Kiel canal, Bosphorus strait and Torres strait.

The straits and canals with convoy times are modeled as a normal port call where the port stay is the time required for the convoy to pass the canal or strait. In the heavily congested canals and straits the speed is restricted within some limits, or even fixed. Both convoy times and speed restrictions need to be modeled correctly as they cannot be added a-posteriori.

Since these convoys depart on a daily basis we introduce a variable $D_{l}$ to denote the time of the day of $t_{l}^{d}$, while the constant $F_{d}$ denotes the frequency of the canal passages in hours (here it is 24 hours). Constraints (17) finds the maximum number of whole days since the start of the week for the departure time at the end port of leg $l$.

$$
0 \leq t_{l}^{d}-F_{w} w_{l}^{d}-F_{d} D_{l}<F_{d}, \quad r \in R, l \in L_{r}
$$

Let $\Gamma$ be the set of different canals and directions and let $S_{\gamma}$ be the set of scheduled convoy times at $\gamma \in \Gamma$, where $\gamma$ is a given canal and direction. The variable $\lambda_{i l}$ is a binary variable indicating if the convoy time $i \in S_{\gamma}$ is used for leg $l$ which goes to a canal (and direction) $\gamma$. The parameter $b_{i \gamma}$ is the time of convoy number $i$ and $S_{\gamma}$ is the set of convoy times for the canal and direction $\gamma \in \Gamma$.

$$
\begin{array}{lr}
b_{i \gamma}-F_{w}\left(1-\lambda_{i l}\right)-t_{l}^{d}+F_{w} w_{l}^{d}+F_{d} D_{l} \leq 0, & r \in R, l \in L_{r}, i \in S_{\gamma}, \gamma \in \Gamma \\
b_{i \gamma}+F_{w}\left(1-\lambda_{i l}\right)-t_{l}^{d}+F_{w} w_{l}^{d}+F_{d} D_{l} \geq 0, & r \in R, l \in L_{r}, i \in S_{\gamma}, \gamma \in \Gamma \\
\sum_{i \in S_{\gamma}} \lambda_{i l}=1 & r \in R, l \in L_{r}, \gamma \in \Gamma
\end{array}
$$

Constraints (18) and (19) ensure that if convoy time $i$ is selected, then the port call time plus the port stay time $P_{l}^{s}$ is the same as convoy time $b_{i \gamma}$. Constraints (20) ensure that exactly one convoy time is selected per leg $l \in L$. The number of canal crossings in a real-life network of a major liner shipping company is discussed in Section 4. 


\subsection{Work shifts}

At some ports the workforce is organized in work shifts. A work shift takes place in a predefined time interval. A day is divided into several of these time intervals. The work shifts influence the cost paid for a port call. The general rule is that a cost must be paid for every work shift overlapped by a port stay. In the case where a work shift is only partly used, a full price is still paid.

To avoid congestion at a port, the port authority often require that vessels must arrive at a start time of a work shift. Such requirements exist at busy ports such as Algeciras, Rotterdam, Bremerhaven, Zeebrucke, Tanjung Pelepas and many others. In the case where a vessel can only arrive at the start time of a work shift then the number of work shifts used during the port call is already minimal. This case can be included in the model by using the constraints (17) to (20) for the port call which is equivalent to the canal passage restriction.

However, when restricting the vessels to arrive at work shift start times, some possible bunker savings may be lost. Thus, we also consider the case were the number of work shifts used by a vessel is reduced by giving a penalty for each work shift touched. Let $U_{l}$ be the set of possible work shifts for the end port call of leg $l$, and let $\hat{U}_{l}$ be the cost of using a single work shift at the end port call of leg $l$. In this case the objective becomes:

$$
\min \sum_{r \in R} \sum_{l \in L_{r}}\left(C_{l}+\hat{P}_{l} m_{l}+\hat{U}_{l}\left(v_{e l}-v_{s l}+1\right)\right)
$$

where $v_{s l}$ is the work shift at arrival, and $v_{e l}$ is the work shift at departure at the end port call of leg $l$.

The following constraints must be added to the problem to ensure that the integer decision variables $v_{s l}$ and $v_{e l}$ are set correctly.

$$
\begin{array}{ll}
\psi_{l} v_{s l}-t_{l}^{d}+F_{w} w_{l}^{d}+\sigma_{l}>0, & r \in R, l \in L_{r}, i \in U_{l} \\
\psi_{l} v_{s l}-t_{l}^{a}+F_{w} w_{l}^{d}+\sigma_{l} \leq \psi_{l}, & r \in R, l \in L_{r}, i \in U_{l} \\
\psi_{l} v_{e l}-t_{l}^{d}+F_{w} w_{l}^{d}+\sigma_{l}>0, & r \in R, l \in L_{r}, i \in U_{l}
\end{array}
$$

where $\psi_{l}$ is the work shift length at the end port of leg $l$ and $\sigma_{l}$ is the time of day of the start of the first work shift at the end port on leg $l$. Arriving earlier in the day than the earliest workshift start time, $\sigma_{l}$, corresponds to work shift number zero. Constraints (22) and (23) find the work shift $v_{s l}$ in progress when the vessel arrives. Constraints $(24)$ combined with the objective ensure that when the penalty $\hat{U}_{l}$ is positive then $v_{e l}$ is the last work shift touched before the departure from the end port of leg $l$ note that all of these fixed times are adjusted with the port stay $P_{l}^{s}$ in order to use the departure time $t_{l}^{d}$. Similar to the case for the canal convoy times, adjusting for these shift cost after a solution is presented may not result in an optimal solution.

\subsection{Emission control areas}

Since 2005 larger emission control areas (ECA) have been introduced starting with the sulphur emission control area (SECA) in the Baltic sea. The ECA requirements often involve restrictions on the amount of $\mathrm{SO}_{x}$ and/or $\mathrm{NO}_{x}$ in the emission. Today several such areas exist both in Europe and along the US and Canadian coastline and in the Caribbean. According to IMO, agreements are in place to ensure that more emission restrictions and areas will be introduced in the future. There are different ways to deal with the emission restrictions in these zones. One is to install scrubbers cleaning the emissions, another is to use bunker types satisfying the emission requirements when sailing in these areas. In this paper we do not consider physical equipment installed or new vessels acquisitions. Hence we only model the costs of different bunker types when a round trip passes through one or more emission control areas.

Entering an ECA usually happens at sea during a journey between two port calls. Clearly, it is cost beneficial to sail slower in an area where more expensive bunker is used and faster outside the area. The difference in speed depends on the cost of the different bunker types. Therefore, in order to optimize cost while respecting the ECA restrictions, it is necessary to find an individual speed for the part of the leg outside the ECA and for the part of the leg inside the ECA. Pseudo port calls are introduced in order to 
split the leg into several parts where each part is a distance of the original leg with a single bunker type requirement. In order to allow for changes in speed, the pseudo port calls at ECA entry/exit points do not imply a penalty when their port call time is changed. This means that ECA zones can be handled by adding extra port calls, without changing the overall model. A specific bunker curve is required for each part of the leg according to the bunker used. Splitting the legs into several sub-legs will lead to a larger model with more decision variables, but significant savings can be achieved by allowing the model to optimize the speed on each sub-leg.

In this way we have a set of legs for each bunker type. $L_{E C A}$ is the set of legs in the ECA zone, and $L_{N E C A}$ is the set of legs not in the ECA zone. The objective function can now be written as:

$$
\min \sum_{l \in L_{E C A}} \Upsilon_{E C A} C_{l}+\sum_{l \in L_{N E C A}} \Upsilon_{N E C A} C_{l}+\sum_{r \in R} \sum_{l \in L_{r}} \hat{P}_{l} m_{l}
$$

where $\Upsilon_{E C A}$ is the cost of bunker used in the ECA zone and $\Upsilon_{N E C A}$ is the cost of the bunker used outside the ECA zone. Note, that only cost is considered here, however, this may not minimize the actual overall emissions but only ensures that the emission requirements are satisfied in the most cost efficient manner. Minimizing the overall emission and pollution is a different topic which is not dealt with here.

\subsection{Piracy areas}

Piracy is a major issue for maritime trade. Some of the primary areas, where piracy is a threat to liner shipping vessels, are the area in the Indian Ocean between the Arabian Peninsular and Somalia, and the area between Malaysia and Indonesia.

There are several ways to deal with a route through a piracy area. The two most relevant ones are convoys protected by armed vessels, or speeding through the piracy area to ensure that pirates are not able to catch up with the vessel.

If convoys are available, they can be modeled by introducing an artificial piracy port call where convoy times can be modeled with constraints (17) to (20) similar to the canal passage time case. In the case where convoys are not used or do not exist it is necessary to sail through the piracy area at a high speed. Thus the leg going through the piracy area should in the same fashion as the ECA bunker requirement have pseudo port calls introduced for entering and leaving the piracy zone. This will split the leg into several parts where each individual part is either inside a piracy zone or outside the piracy zones. For the parts of the leg inside the piracy zone a minimum speed requirement is introduced. Given a leg $l$ which is in a piracy zone and a maximum time $T_{l}^{x}$ the constraint required to be satisfied for leg $l$ is represented in

$$
t_{l}^{a}-t_{l^{\prime}}^{d}+F_{w} S_{r} f_{l} \leq T_{l}^{x}, \quad r \in R,\left(l^{\prime}, l\right) \in L_{r}
$$

Note that by setting $\hat{P}_{l}$ for the pseudo port calls to zero, then the piracy port call times will not be penalized in the objective (2) when changed.

\subsection{Penalizing longer and rewarding shorter transit times}

Until now we have assumed that transit time constraints (12) are hard limits. We now relax these constraints, and instead penalize delays in the objective. This reflects that if transit times are increased, then either customers will be lost, or lower freight rates can be achieved. The penalty is defined by a scalar $\eta^{q} \geq 0, q \in Q$ for the delay of the demand. There will, however, still be a limit on how much delay is allowed. We will also introduce a bonus parameter $\zeta^{q} \geq 0$ and $\zeta^{q}<\eta_{r}^{q}$ for shortening the transit time.

The transit time constraints (12) is then changed to

$$
\sum_{c=(l, h) \in O_{q}}\left(t_{h}^{a}-t_{l}^{d}+H_{c}+F_{w}\left(w_{l}^{d}+x_{c}\right)+\sum_{l, l^{\prime} \in L_{q}, l, l^{\prime} \in L_{r}, r \in R}\left(t_{l}^{d}-t_{l^{\prime}}^{d}+F_{w} S_{r} f_{l}\right)-P_{q}^{s}+e^{q}-b^{q}=D_{q}, \quad q \in Q,\right.
$$

where $e^{q}, b^{q} \geq 0$ are continuous variables denoting how much earlier/later the demand arrives compared to the transit time. It can be relevant to ensure that $e^{q}$ is always within a specific interval. This can be ensured by introducing an upper bound on $e^{q}$. Note that there should never be an upper bound on $b^{q}$. The objective is then changed to 


$$
\min \sum_{l \in L_{E C A}} \Upsilon_{E C A} C_{l}+\sum_{l \in L_{N E C A}} \Upsilon_{N E C A} C_{l}+\sum_{r \in R} \sum_{l \in L_{r}} \hat{P}_{l} m_{l}+\sum_{q \in Q}\left(\eta^{q} b^{q}-\zeta^{q} e^{q}\right)
$$

Softening the time constraints enables a proper trade-off between bunker costs and delay costs. Moreover, the objective function makes it possible to prioritize the arrival time of different demands by differentiating the penalties $\eta^{q}, \zeta^{q}$.

\subsection{Integrating feeder services with different frequencies}

Major liner shipping companies frequently cooperate to offer as many origin-destination products as possible. Most major companies use a weekly frequency on their services. However, some feeder services visiting smaller ports may be using a bi-weekly frequency. Moreover, the Panama railway may be used to transport cargo between the Pacific port and the Caribbean port with departures twice a day. Feeder services, used for end delivery of containers, but not operated by the studied company, are called foreign feeder services. It is easy to incorporate the schedules of collaborating companies if they use the same frequency as the optimized company. However, if the frequency of two services differ, then the model must be able to accommodate the disparity. The schedules operated by other companies are assumed to be fixed. On the other hand transit time satisfaction must be assured and even though the schedules of collaborating companies may not be changed, it is important that the schedules are correctly modeled. As noted by Wang et al. [35] services with a different frequency can be modeled by repeating the service until it is divisible by the frequency of the service belonging to the optimizing liner shipping company. Since the schedule is not changed in [35] the problem solved is not the same as studied here. Moreover, as noted in Section 2, services of different frequency were not included in the test cases in [35]. The modeling of transit time for demands using services with different frequencies is not simple since each leg is represented by several edges in the graph formulation, and it is important to know which edge is used for the transshipment in order to calculate transit times correctly.

There are different scenarios for the form of collaboration on the demand and the relation between the frequency of the collaborating services:

1 The frequency of the optimized service is divisible by that of the collaborating service, and the collaborating service is used for

(a) the last part of the journey ending at the destination.

(b) the first part of the journey starting at the origin.

(c) a middle part of the journey which is neither the first or last part.

2 The frequency of the collaborating service is divisible by that of the optimized service and (a),(b) or (c).

Let the set of copies of an leg $l$ on a service be $M_{l}$. Let $\alpha_{l^{\prime}}^{c}$ be a binary variable where $c=(k, l) \in O$ is a connection and $l^{\prime} \in M_{l}$ is a leg copy of the leg $l$. Let inequalities (29) ensure that only one of the leg copies is selected for a connection:

$$
\sum_{l^{\prime} \in M_{l}} \alpha_{l^{\prime}}^{c}=1, \quad c=(k, l) \in O, c=(l) \in O^{\prime}, l \in L_{r}, r \in R^{\prime},
$$

where $O^{\prime}$ is the connections which simply involves the loading at origin and $R^{\prime}$ is the set of foreign services with a frequency which is a divisor of the frequency of the optimized services.

In case (1a), where the foreign service is the last service on the journey of the demand and the frequency of the optimized service is divisible by the foreign service, the first feasible leg on the foreign service should be selected in order to provide the minimum transit time for the journey thus reducing the penalty or increasing a reward. Therefore the constraint ensuring feasibility in the selection of the leg $l$ on the foreign feeder in a connection $c=(k, l)$ where $l$ is on the foreign feeder can be represented as

$$
\begin{array}{r}
\sum_{l^{\prime} \in M_{l}}\left(\left(t_{l^{\prime}}^{a}-F_{w} w_{l^{\prime}}^{a}\right) \alpha_{l^{\prime}}^{c}\right)-t_{k}^{d}-H_{c}+F_{w}\left(w_{k}^{d}+K_{c}^{w}+x_{c}+a_{c}\right) \geq 0, \\
q \in Q^{\prime}, c=(k, l) \in O_{q}, l \in L_{r}, r \in R^{\prime}, k \in L_{s}, s \in R
\end{array}
$$


where $Q^{\prime}$ is the set of demands for which the last service on the demand journey is in $R^{\prime}$. By definition the binary variable $a_{c}$ is related to leg $k$ (see constraint (9)). Note that if the duration of foreign service $r$ is also a divisor of the frequency of $s$ then the term $F_{w} w_{l^{\prime}}^{a}$ can be removed from inequality (30). Otherwise $w_{l^{\prime}}^{a}$ is the number of whole weeks from time zero to the time $t_{l^{\prime}}^{a}$. The variable $w_{l^{\prime}}^{a}$ is equal to $w_{l^{\prime}}^{d}$ if $t_{l^{\prime}}^{a}-F_{w} w_{l^{\prime}}^{d}>0$ otherwise $w_{l^{\prime}}^{a}$ is equal to $w_{l^{\prime}}^{d}-1$ under the realistic assumption that a vessel never has a port stay of duration longer than a week.

In the case (1b) the foreign service is the service $s \in R^{\prime}$ used from the origin of the demand. Thus the leg $l \in L_{s}$ used on the departure from origin determines the leg $g \in L_{s}$ arriving at the first connection. This means that for the connection $c=(g, h)$ the selection of the foreign feeder leg at origin $l^{\prime} \in M_{l}$, where $c=(l), l \in L_{s}$, determines the leg $g^{\prime} \in M_{g}$. Thus we define a variable $\beta_{g^{\prime}}^{c}$ which is equal to the variable of the corresponding leg $\alpha_{l^{\prime}}^{c}$ for loading on the foreign feeder service $s$.

Now the constraint ensuring feasibility in the selection of the leg $g^{\prime} \in M_{g}$ on the foreign feeder in a connection $c=(g, h)$, where $h$ is on the optimized services, can be formulated as:

$$
\begin{array}{r}
t_{h}^{a}-F_{w} w_{h}^{a}-\sum_{g^{\prime} \in M_{g}}\left(\left(t_{g^{\prime}}^{d}-F_{w}\left(w_{g^{\prime}}^{d}\right)\right) \beta_{g^{\prime}}^{c}\right)-H_{c}+F_{w}\left(K_{c}^{w}+x_{c}\right) \geq 0, \\
q \in Q^{\prime \prime}, c=(g, h) \in O_{q}, h \in L_{r}, r \in R, g \in L_{s}, s \in R^{\prime},
\end{array}
$$

where $Q^{\prime \prime}$ is the set of demands where the first service on the journey is a foreign service in $s \in R^{\prime}$. As a result of the assumption that service $s$ is a foreign service and thus the departure and arrival times of the leg are fixed by an other operator, then $t_{g^{\prime}}^{d}$ and $w_{g^{\prime}}^{d}$ are fixed for every leg $g^{\prime}$ on $s$. Again, the term $F_{w} w_{g^{\prime}}^{d}$ can be removed from inequality (31) if the duration of foreign service $s \in R^{\prime}$ is also a divisor of the frequency of $r \in R$.

\section{Panama rail service}

- . - - Service in the Pacific

$\bullet \bullet \bullet \bullet \bullet$ The container path from Pacific to Caribbean

.......... Service in the Caribbean

(a) Definitions

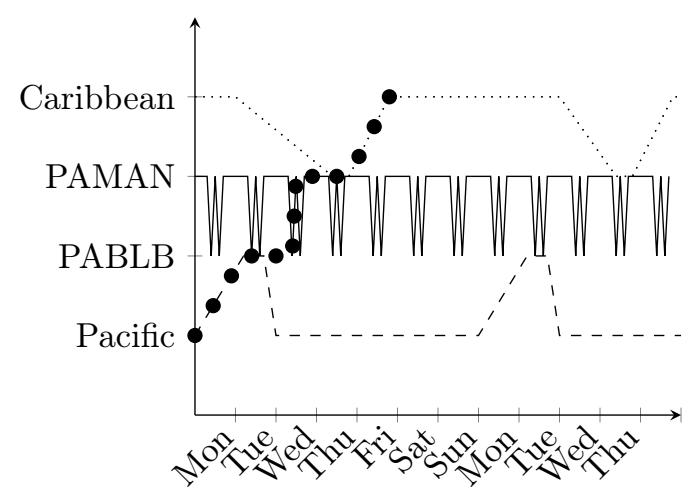

Time



Time

(b) Initial schedule

(c) Changed schedule for the Pacific service

Figure 2: The journey of a demand using a foreign service with a different frequency. The foreign service is the Panama rail service which has a daily frequency which is a divisor of weekly frequency of the two remaining services.

For the case (1c) we let the set $Q^{\prime \prime \prime}$ contain the demands with paths where the foreign service is neither at origin nor destination and the frequency of the foreign service is a divisor of the optimized services. An example of a journey in $Q^{\prime \prime \prime}$ using a foreign service is shown in Figure 2 where a missed connection is shown in Figure 2(c). To solve the problem we use the constraints (29), (30) and (31) replacing the set $Q^{\prime}$ in constraints (30) and the set $Q^{\prime \prime}$ in constraints (31) with the set $Q^{\prime \prime \prime}$. 
In case 2 we assume that the foreign feeder has a frequency which is divisible by the frequency of the optimized services. For cases (2a) and (2b) nothing needs to be changed as it can be assumed that the demand is departing from the origin at the frequency of the foreign service. Keeping the demand at the origin is always better than paying for storage at a port. Thus in (2a) the demand would only depart from the origin at the frequency of the foreign feeder at the destination in order to avoid unnecessary storage at a port during the journey. For case $(2 \mathrm{~b})$ the logic is even simpler as it is assumed that the demand will arrive at port for the scheduled departure of the foreign feeder, thus it will arrive at the frequency of the foreign feeder. This observation also holds for case (2c) where the foreign service is neither at the start nor at the end of the journey. In case (2c) the departure from the origin is therefore assumed to always match the frequency of the foreign service that demands are transshipped to. Thus the connection $c=(k, l)$ and $l \in R^{\prime \prime}$, where $R^{\prime \prime}$ contains the foreign services of a frequency which is divisible by the optimized services, can be handled by constraints (9) to (11) in the original model. The connection $c^{\prime}=(g, h)$ transferring from the foreign service to an optimized service on a journey in case (2c) can also be handled by the constraints (9) to (11) as the service connecting to has the frequency used in the modeling.

The time used connecting to and from a foreign service of different frequency must be included correctly to ensure that the transit time is satisfied. Therefore constraint (27) must be altered to include the transit times of the cases (1a) and (1b). In the constraint (27) the term

$$
\sum_{c=(k, l) \in O_{q}}\left(t_{l}^{a}-t_{k}^{d}-F_{w}\left(w_{l}^{d}-w_{k}^{d}-x_{c}-K_{c}^{w}\right)\right)
$$

represents the connections and thus this term must be expanded so that the new situations are handled. Note that the duration of the sailing time on the foreign feeder is the same for all pairs $j^{\prime} \in M_{j}, j \in L_{r}$ where $r \in R^{\prime}$ and corresponding leg $i^{\prime} \in M_{i}, i \in L_{r}$ and that a specific $i^{\prime}$ corresponds to each $j^{\prime}$. The term

$$
\sum_{i, j \in L_{q}, i, j \in L_{r}, r \in R}\left(t_{i}^{d}-t_{j}^{d}+F_{w} S_{r} f_{i}\right)
$$

in constraint (27), can be written as:

$$
\sum_{i, j \in L_{q}, i, j \in L_{r}, r \in R^{\prime}, c \in O_{q}}\left(\sum_{i^{\prime} \in M_{i}} t_{i^{\prime}}^{d} \beta_{i^{\prime}}^{c}-\sum_{j^{\prime} \in M_{j}} t_{j^{\prime}}^{d} \alpha_{j^{\prime}}^{c}+F_{w} S_{r} f_{i^{\prime}}\right)
$$

for the case where $i$ and $j$ are on a service $r \in R^{\prime}$. However, since the sailing time is fixed we will just use the term in equation (33) where $i$ represents the earliest leg in the set $M_{i}$ and $j$ is the corresponding leg in the set $M_{j}$ and $i, j \in L_{r}, r \in R \cup R^{\prime}$. Thus the transit time constraint (27) will look as follows:

$$
\begin{aligned}
& \quad \sum_{c=(k, l) \in O_{q}, l, h \in R}\left(t_{l}^{a}-t_{k}^{d}-F_{w}\left(w_{l}^{d}-w_{k}^{d}-x_{c}-K_{c}^{w}\right)\right. \\
& +\sum_{c=(k, l) \in O_{q}, k \in R, l \in R^{\prime}}\left(\sum_{l^{\prime} \in M_{l}}\left(t_{l^{\prime}}^{a}-F_{w} w_{l^{\prime}}^{d}\right) \alpha_{l^{\prime}}^{c}\right)-t_{k}^{d}+F_{w}\left(w_{k}^{d}+x_{c}+K_{c}^{w}\right) \\
& +\sum_{c=(g, h) \in O_{q}, g \in R^{\prime}, h \in R}\left(t_{h}^{a}-\sum_{g^{\prime} \in M_{g}}\left(\left(t_{g^{\prime}}^{d}-F_{w} w_{g^{\prime}}^{a}\right) \beta_{g^{\prime}}^{c}\right)-F_{w}\left(w_{h}^{a}-x_{c}-K_{c}^{w}\right)\right) \\
& \quad+\sum_{i, j \in L_{q}, i, j \in L_{r}, r \in R \cup R^{\prime}}\left(t_{i}^{d}-t_{j}^{d}+F_{w} S_{r} f_{i}\right)-P_{q}^{s}+e^{q}-b^{q}=D_{q}, \quad q \in Q,
\end{aligned}
$$

Since most liner shipping companies operate with a weekly frequency it is not very common to see mathematical models linking several frequencies. The importance of handling this situation is discussed in Section 4.7 .

\subsection{Vessel Sharing Agreements}

Vessel sharing agreements (VSA) make it possible for two or more operators to share a service and thereby profit from economies of scale. In a VSA each operator will have the right to use a given share of 
the vessel capacity, hence the bunker savings on the service will be scaled according to this share. This is easily incorporated in the model by reducing the bunker curve for that service so that it matches the share of the vessel.

Another challenge when speed optimizing services with VSA is that transit times are only known for demands of the present operator and not for the demands of collaborators. Moreover, the collaborating operators may have contradicting wishes for the arrival times at ports and hence the bunker consumption. We have therefore chosen to fix the berthing times of the VSA services, since no parties will be affected negatively. Unfortunately this would limit the bunker saving potential. Therefore it is recommended that a liner shipping company thoroughly investigates the lost savings when using the fixed schedule option by systematically fixing and opening these VSA port calls according to the known demand flow and using the achieved information as a basis for negotiation. Knowing the possible bunker savings which can be obtained by rescheduling a port call will provide an important input to the VSA companies when negotiating changes on the services.

\subsection{Robustness}

Next to bunker consumption, robustness of routes, is one of the most important needs of a liner shipping company. If the network is not robust, delays will occur, and delays may propagate throughout the network in a cascade effect. This implies delayed cargo, but also increased bunker consumption, since vessels have to sail faster to catch up.

In our collaborating shipping company, the robustness of a leg is defined as the slack time on a leg, i.e., the gap between the planned sailing time and the fastest possible sailing time (at maximum speed). In other words, the robustness is defined by how much time can be caught up by sailing at maximum speed. Short legs are generally more vulnerable for delays, since there is not much time to catch up even when sailing at maximum speed. This measure of robustness is similar to the measure used in [36] for aircraft routing and crew scheduling. A schedule is denoted operationally robust if it evades critical legs, where a leg is critical if the slack time is below a given threshold value.

Currently, robustness is in major liner shipping companies obtained by adjusting the berthing times manually at ports where delays occur frequently. However, a manual approach may create new bottlenecks at other ports in the network, as well as leading to increased bunker consumption. We will therefore present a unified model, considering the whole network, where bunker consumption and robustness are two independent objectives.

To be more formal, let $\rho_{l} \geq 0$ be the robustness of leg $l \in L$. We can determine $\rho_{l}$ by modifying equation (4) to the following form

$$
t_{l}^{a}-t_{l^{\prime}}^{d}+F_{w} S_{r} f_{l}-\rho_{l}=T_{l}, \quad r \in R,\left(l^{\prime}, l\right) \in L_{r}
$$

Notice that $\rho_{l}$ serves as the slack variable in the constraint. Since we wish to maximize the robustness of the network, one could aim at maximizing $\sum_{l \in L} \rho_{l}$. This may, however, lead to a very uneven distribution of the robustness, so we go for a better formulation, where we wish to ensure a minimum robustness $\mu$ at every leg. In this way we get the constraint

$$
\rho_{l} \geq \alpha_{l} \mu, \quad l \in L
$$

The parameter $\alpha_{l}$, where $0 \leq \alpha_{l} \leq 1$, can be used by the shipping company to weigh the importance of robustness at different legs. For instance, if $\alpha_{l}=1 / 2$ for a given leg $l$, then then only half as much slack time is needed as on the other legs. The importance of a leg may depend on the degree of congestion in the destination port, or the size of the vessel.

Our problem now becomes a bi-objective optimization problem where the original objective (2) is replaced by

$$
\min _{\max } \sum_{r \in R} \sum_{l \in L_{r}}\left(C_{l}+\hat{P}_{l} m_{l}\right)
$$


The $\epsilon$-frontier is found by using the $\epsilon$-constrained method developed by Haimes et al. [17]. For further details on the $\epsilon$ - efficient frontier and multi-objective optimization the readers are referred to Carmichael [6], White [37] and Ehrgott and Gandibleux [11].

As a first step, we remove objective (39) and minimize the bunker consumption. This will give us an optimal operational cost $z^{\prime}$ and a corresponding value of robustness $\mu^{\prime}$. We then add the constraint $\mu \geq \mu^{\prime}+\epsilon$ and solve the problem again. The process is repeated until the solution $\left(z^{\prime}, \mu^{\prime}\right)$ is less than $\epsilon$ from the optimal value of $\mu$. The pairs $\left(z^{\prime}, \mu^{\prime}\right)$ form the Pareto-optimal frontier, making it possible for the decision maker to find a proper trade-off between operational costs and robustness.

\section{Tests and results}

In this section the different business constraints are studied and their effect on bunker consumption and solution time is reported. The detailed study uncovers the potential for fuel saving, and identify which business constraints currently are the limiting factor.

The test sets were designed in collaboration with a major liner shipping company. They are based on detailed data, some of which are publicly available while other parts contain confidential data provided by the company. For each leg the company has provided a leg specific bunker curve and cost curve. These curves are based on historic performance of the vessels, and historic bunker prices for the specific leg. The data contains the schedule of the company at the time provided this is referred to as the original schedule. The test sets have been validated by the liner shipping company.

The model is solved using CPLEX and the model and corresponding data have been generated using $\mathrm{C}++$. All tests have been conducted on a shared 10 core Intel Xeon E5-2660v3 -2013 Huawei XH620 V3 with 128 GB memory.

The studied test instances are all based on one overall network, where different subsets are selected for studying local optimizations or selected constraints.

\begin{tabular}{|l|r|}
\hline \multicolumn{2}{|c|}{ Network size } \\
\hline services & 142 \\
legs & 2170 \\
demands & 20830 \\
connections & 3892 \\
\hline
\end{tabular}

Table 2: Characteristics of the overall network studied

\begin{tabular}{|l|r|r|r|r|r|}
\hline instance & services & port calls & remands & connections & legs \\
\hline ECA-service & 1 & 39 & 709 & 181 & 39 \\
Single-service 1 & 1 & 26 & 736 & 112 & 26 \\
Single-service 2 & 1 & 23 & 1285 & 201 & 23 \\
Midsize-Africa & 3 & 39 & 890 & 206 & 39 \\
Midsize-US & 3 & 69 & 1327 & 223 & 69 \\
Two-services 1 \& & 2 & 49 & 1906 & 302 & 49 \\
Europe-Asia services & 8 & 223 & 8331 & 1403 & 223 \\
Latin America services & 14 & 244 & 5077 & 826 & 244 \\
One-operator & 66 & 1146 & 19572 & 3579 & 1146 \\
\hline
\end{tabular}

Table 3: Test instances used in the computational experiments. Each instance considers a subsets of the network presented in Table 2, where the remaining services are kept fixed.

Table 2 shows the characteristics of the overall network studied. The network also includes services operated by other companies. These connecting services may be used to flow the demand, but the speed and port call times of the services may not be changed. Each demand represents a route from an origin to 
a destination with a given amount of containers. Since the route of all demands remains unchanged, then there is sufficient capacity to flow all demands.

Based on the network described in Table 2 the collaborating company has proposed 7 of the instances described in Table 3 and we have constructed two instances to show more midsize cases. The instances are constructed by selecting a subset of the overall network, while surrounding services are kept fixed. The services in instance Single-service 1 and Single-service 2 are selected so that there are several connections between the two services. Test instance Two-services 1 \& 2 contains the two services and all connections with port calls on one or both of the services. The instances Midsize-Africa and Midsize-US consists of 3 services visiting overlapping ports in Africa and US respectively. The Africa ports often do not have work shift requirements as opposed to the US. Although we have not looked into the demand flow, one could expect different behavior of demand flowing through the Africa services and the US services. The test instances Europe-Asia services and Latin America services each contain a set of regional services. These two instances represent current practice of regional service managers with responsibility for the schedule of services within their region and are considered the largest relevant instances. The last test instance One-operator is the entire network of a large liner shipping company including services using vessel sharing agreements. It is unlikely that the entire network would be rescheduled at once, however, it is relevant to show that the model also can be solved for the extreme case. The test cases shown in Table 3 include canals, ECA areas, piracy areas, delay penalties, work shifts and services of different frequencies, and hence form the basis for our experiments. They will be used for testing the individual business constraints.

Since the test cases are based on adapted real-life cases it is for confidentiality reasons not possible to publish the optimal solutions and costs. Due to the focus on CO2 emission we have chosen to only report the reduction in bunker consumption satisfying the operational rules defined by the the liner shipping company. Therefore we report the percentage of reduction in bunker consumption from the original schedule. Since we are provided with the original schedule then this schedule is used as a warm-start in all tests. The penalty and bonus values were defined by the company. Note that the bunker savings are only calculated for the optimized legs in the test instance and not for the entire network.

As the complete network contains all business constraints, such as canals, piracy, ECA zones, different frequency feeders and workshifts, all tests have been conducted with the full set of business constraints. However, since some of the instances only optimize a subset of the services, not all business constraints may apply for a given instance, as discussed in the following sections. The delay penalty and early bonus is included in the tests when the slack is above 48 hours or no slack value is provided. There is always an upper limit on the transit time delay of 30 days.

The computational experiments are structured as follows. First, in Section 4.1 and 4.2 we study the complete instances using soft time constraints. Next, in Section 4.3 to 4.7 we analyze the individual business constraints in more details. Finally, in Section 4.8 we report results for planning robust schedules. Moreover, we present the full $\epsilon$-frontier for some of the instances when solving the problem as a bi-objective optimization problem.

\subsection{Late penalty and early bonus}

Shipping companies have different priorities in terms of delay, depending on whether the cargo is refrigerated, or the customer has high priority. To reflect these priorities each demand has an individual penalty as described in Section 3.7. In the tests shown in Table 4, the penalty is a set to $100 / 24 \$$ per hour delay per FFE (forty foot equivalent container unit), and the bonus for arriving early is $10 / 24 \$$ per hour per FFE. All of the business requirements are being used in the tests presented in Table 4 and Figure 3 .

The savings reported in column three of Table 4 are the bunker savings. The bunker reduction is reported as percent savings compared to the bunker costs of the original schedule. The savings are only compared to the original bunker cost for the optimized services, ignoring the fixed part of the network. It should be noted that a warm-start consisting of the original schedule was used on all instances. Even though using a warm-start did provide better solutions within the first seconds then it does not affect the final result reported. After an hour the optimality gaps were for all instances less than $1.8 \%$ and the bunker savings were for all instances above $3.5 \%$.

The penalties for delay are included in the objective but not presented in the table. In Figure 3 the distribution of the new transit times for the demand is shown for the One-operator instance of Table 4. 


\begin{tabular}{|l|r|r|r|r|}
\hline instance & Port calls changed & Savings \% & Time $(\mathrm{s})$ & Gap \\
\hline Single-service 1 & 18 & 8.42 & 615 & Optimal \\
Single-service 2 & 14 & 7.28 & 83 & Optimal \\
Two-services 1+2 & 32 & 7.63 & 3600 & 1.77 \\
Midsize Africa & 15 & 11.20 & 3600 & 1.19 \\
Midsize US & 19 & 4.12 & 3600 & 1.54 \\
Europe-Asia services & 145 & 3.60 & 3600 & 0.99 \\
Latin America services & 161 & 4.34 & 3600 & 1.14 \\
One-operator & 616 & 3.78 & 3600 & 1.72 \\
\hline
\end{tabular}

Table 4: The bunker savings achieved when the transit time is provided a slack of 30 days and delay penalty from original transit time depending on the size of demand. An optimal solution is denoted by "Optimal".

The test has been run with CPLEX given a time limit of one hour.

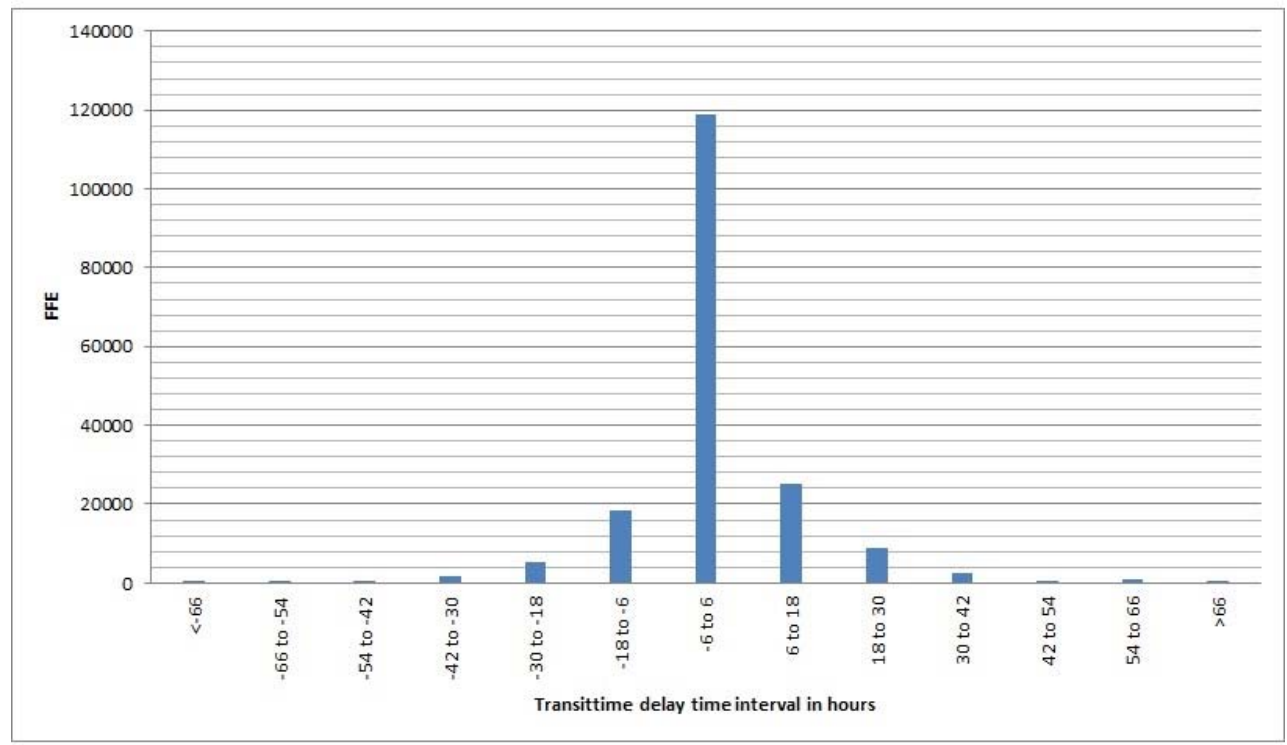

Figure 3: Histogram of freight delay distribution on the One-operator instance. The number of FFE is depicted as function of the arrival time (deviation from transit time)

Figure 3 shows the distribution of delays for the solution of the One-operator test instance of Table 4. It is seen that most of the demand is not delayed significantly. Moreover, the amount of demand arriving later is somehow symmetric to demand arriving earlier, with a slight tendency to spread out the the early arrivals more than the late arrivals. Increasing the penalty to $\$ 100$ for each hour delay and $\$ 10$ for each hour earlier arrival, makes the delay distribution more symmetric.

\subsection{CPLEX tuning}

Since the bigger instances still have a gap of 1-1,5\%, we investigated whether proper tuning of CPLEX parameters could close this gap. We have tested several different settings among others we have tested the relaxation induced neighborhood search which is a primal heuristic provided by CPLEX for the cases with more than one service the use of the heuristic shows a small improvement in the solution value. We have through preliminary testing selected to run the heuristic every 500 or 700 nodes depending on the instance. We use the default CPLEX cut generations as we found that this performs quite well. Also the MIP emphasis parameter of CPLEX has been tested and although emphasizing finding hidden feasible solutions 
improved the solution and optimality gap for some instances it did the opposite for other instances and thus this selection would not be recommended as a default for the model. It should be noted that for all instances except One-operator good solutions were found within the first 5 minutes and the remaining time was used mainly on closing the optimality gap all though slight improvements of the best found solution did take place in half of the instances.

\subsection{ECA regulations}

In the data instances presented in Tables 2 and 3 the ECA and non ECA part of a direct trip is represented by splitting the leg into two parts by adding a virtual port call (without any change penalty) when crossing the ECA border. In order to evaluate the effect of selecting different speeds on the parts inside and outside ECA zones the instance ECA-service from Table 3 is used. This service has 39 port calls, however, 4 of these are virtual port calls at sea when crossing the ECA border (11\% of the legs in the instance). Thus a version of the ECA-service with only 35 port calls excluding the virtual port calls was generated. In the new version the different segments of a direct trip was merged in order to insure a fixed speed on the different segments so that the secant bunker approximation could be applied for the entire leg. If a service does not have a leg crossing the boarder of an ECA zone then the solution would remain the same. Thus a test case of a single service with four legs crossing the border of the ECA has been selected from the network in Table 2. The company has the last few years introduced slow steaming in the ECA zones. Thus to complete this test we have combined the leg consumption and time of ECA entering legs into one leg. The costs used for the two different fuel types is an average cost estimated by the company providing data and is representative of the actual cost occurred. Clearly these costs can change over time due to fluctuations in fuel prices. Only the four legs crossing the border of the ECA zone are split into two parts, since the optimal choice for the remaining legs is a constant speed on the whole leg.

If the model has to use the same speed for a leg crossing ECA and non ECA, we were able to achieve $2.84 \%$ savings. In case we allow different speeds for the two sub-legs, $3.05 \%$ savings was achieved. The difference between these two solutions would correspond to additional savings of around 400000 dollars yearly. The difference is not only in the cost but there is also a slight difference in the overall time used on the legs crossing the ECA border. The instance One-operator contains in total 81 legs which cross the border of an ECA, this corresponds to $7 \%$ of the legs on the network. The additional savings obtained were $0.2 \%$, which can be achieved by simple changes not adding significant complexity to the problem. The tests presented in Table 4 allow the selection of different speeds in the different zones on legs crossing the border of an ECA zone. The benefits from this optimization is a reduced fuel consumption in the ECA zones thus reducing the pollution in the ECA zones even further. However, this also results in larger fuel consumption outside the ECA zone. There is an ongoing discussion on whether ECA zones is the best way of reducing emission from vessels, as described in [23].

\subsection{Piracy areas}

The piracy areas are considered in the test presented in Table 4 and Figure 3. Since the minimum speed required in the piracy areas is quite high this requirement will increase the overall bunker cost.

In the test instance One-operator, a total of 79 legs cross a piracy area, which corresponds to $6 \%$ of the total number of legs. This confirms the importance of correctly modeling this business constraint.

\subsection{Canal convoy times}

The canal convoy times are very important for liner shipping companies operating in both Europe and Asia or South America and the Caribbean.

\begin{tabular}{|l|l|c|}
\hline Name & Country & Services passing canal \\
\hline Suez Canal & Egypt & 15 \\
Panama Canal & Panama & 4 \\
\hline
\end{tabular}

Table 5: Canals in the test network and the number of services in the test network traversing each canal. 
Table 5 shows the canals included in the test network and the number of services visiting each canal in the One-operator test instance. The table shows that $28.8 \%$ of the services in the One-operator test instance pass through one of the two canals. The Suez canal is passed by $22.7 \%$ of the services and the Panama canal is passed by $6.1 \%$ of the services. The total number of times a canal is traversed on the Oneoperator test instance is 38 . This is $3.3 \%$ of the port calls in the test instance, which is a quite considerable part of the network. For both canals there is only one meeting time a day, therefore missing such a meeting time would result in up to 24 hours delay which would have to be caught up on the following leg, resulting in increased bunker consumption. There are other less frequently visited canals such as Torres Strait, Kiel Canal, Canakkale and Bosphorus (not considered here) which may also have canal passing time constraints. The canal convoy times are included in the test case for work shifts shown in Tables 7 and 8. The canal convoy times are also included in the test results reported in Table 4 and Figure 3.

\subsection{Work shifts}

In the test instance One-operator, 138 out of 1146 port calls include work shifts. This corresponds to $12.1 \%$ of the port calls, which is a quite significant part of the network. The ports with work shifts have 3 to 5 work shifts in a day. In Section 3.4 two different work shift constraints were proposed:

(a) The port call must begin at the start of a work shift

(b) A fee is paid for every work shift a port call touches

Clearly, the first constraint (a) will minimize the direct fees paid but it may result in an overall higher bunker consumption as our speed choices are more restricted by (a) than by (b). To evaluate the difference in bunker consumption between the two versions of the work shift constraints, the two models are compared for a selected single service with 21 port calls and 273 open connections and 1403 demands using the service. On the selected service 2 port calls are canals and 5 port calls have work shifts. Thus $23.8 \%$ of the port calls on the selected service include work shifts. In Table 6 the port calls with work shift times and the canal convoy calls of the selected service are shown. In column two the sequence number of the port call on the service is shown. Note that there are port calls without work shift rules between some of the port calls shown. The sixth column shows the start time for the earliest work shift and column seven provides the length of each work shift. The port calls which do not satisfy the work shifts in the original schedule are marked with an asterisk "*".

\begin{tabular}{|l|r|r|r|r|r|r|}
\hline Instance & Call number & Day & Hour & Stay & WS start & WS length \\
\hline Suez Canal* & 8 & 33 & 1 & 16 & 2 & 24 \\
Algeciras & 9 & 38 & 14 & 18 & 2 & 6 \\
Rotterdam* & 10 & 42 & 15.5 & 31.5 & 7 & 8 \\
Bremerhaven & 11 & 44 & 22 & 32 & 6 & 8 \\
Rotterdam & 13 & 47 & 23 & 26 & 7 & 8 \\
Algeciras & 16 & 55 & 14 & 12 & 2 & 6 \\
Suez Canal & 17 & 60 & 19 & 22 & 19 & 24 \\
\hline
\end{tabular}

Table 6: The port calls which either have work shift or canal convoy time constraints on an original schedule for a service with 21 port calls . The remaining ports on the service have no restriction. "Call number" denotes the sequence number of the port in the sequence of 21 port calls. The port calls marked with "*" are not visited on their work shift times or convoy times in the original scheduled.

In Table 7 the savings achieved using the two work shift models are reported together with the savings obtained by excluding workshift constraints. The table shows that without work shift constraints it is possible to achieve savings of $3.99 \%$. Minimizing the number of work shifts reduces the savings to $3.66 \%$. Always calling a port at the beginning of a work shift further reduces the savings to $3.57 \%$. Thus, the reduction in savings is generally small when introducing relaxed work shift constraints compared to the tighter ones. Significant cost reductions can be achieved even when respecting the tight constraints restricting port call times to the start of the workshifts. 


\begin{tabular}{|l|r|r|r|r|}
\hline Model case & slack & Changed & savings & time (s) \\
\hline No work shift & 48 & 13 & $3.99 \%$ & 0.96 \\
Work shift optimized & 48 & 13 & $3.66 \%$ & 1.16 \\
Work shift fixed & 48 & 11 & $3.57 \%$ & 0.42 \\
\hline
\end{tabular}

Table 7: The cost reductions achieved on the service without any work shift constraints, and each of the two work shift models. In the test a 48 hours slack is allowed.

\begin{tabular}{|l|r|r|r|}
\hline Instance & call number & ws fixed (h) & ws optimize (h) \\
\hline Suez Canal* & 8 & +25 & +25 \\
Algeciras & 9 & +6 & +6 \\
Rotterdam* & 10 & +7.5 & +7.5 \\
Bremerhaven & 11 & 0 & 0 \\
Rotterdam & 13 & 0 & -1 \\
Algeciras & 16 & 0 & 0 \\
Suez Canal & 17 & 0 & 0 \\
\hline
\end{tabular}

Table 8: New port call times using arriving at work shift start requirement versus the requirement of minimizing the number of work shifts used

In Table 8 the new schedules are shown for the two different work shift requirements. It is interesting to notice that the only difference between the two schedules is that the version minimizing the number of work shifts used, speeds up on the leg to Rotterdam (port call number 13) thus arriving one hour early. This results in extra time available for the legs between Rotterdam and Algeciras. Even though it is shown in table 7 that additional savings can be achieved by minimizing the number of work shifts used the real-life liner shipping operations use the requirement of arriving at the start of a work shift due to port restrictions. This is therefore also the work shift model used in the tests reported in Table 4.

\subsection{Daily service Panama Rail}

In the network presented in Table 2 the only service operated at a different frequency is the Panama rail service. This service is operated twice a day. Since the frequency of the optimized service is divisible by the frequency of the Panama rail this can be represented as case 1 (a), (b) and (c) in Section 3.8. Most of the demands in the test network can be represented by the case 1(c). Table 9 reports for each test instance the number of demands using the Panama Rail service. For the Latin America services $8.3 \%$ of the demand makes use of the Panama rail.

\begin{tabular}{|l|r|}
\hline instance & Panama Rail demands \\
\hline Single-service 1 & 0 \\
Single-service 2 & 0 \\
Two-services 1 \& 2 & 0 \\
Midsize-Africa & 0 \\
Midsize-US & 0 \\
Europe-Asia services & 12 \\
Latin America services & 422 \\
One-operator & 424 \\
\hline
\end{tabular}

Table 9: The number of demands using the Panama rail in each test instance.

The constraints modeling the daily service of the Panama rail make the problem more complex to solve. The more demands are transported through the Panama rail service, the harder the problem becomes to solve, as numerous auxiliary variables are introduced to model the flow. There are currently 14 departures 


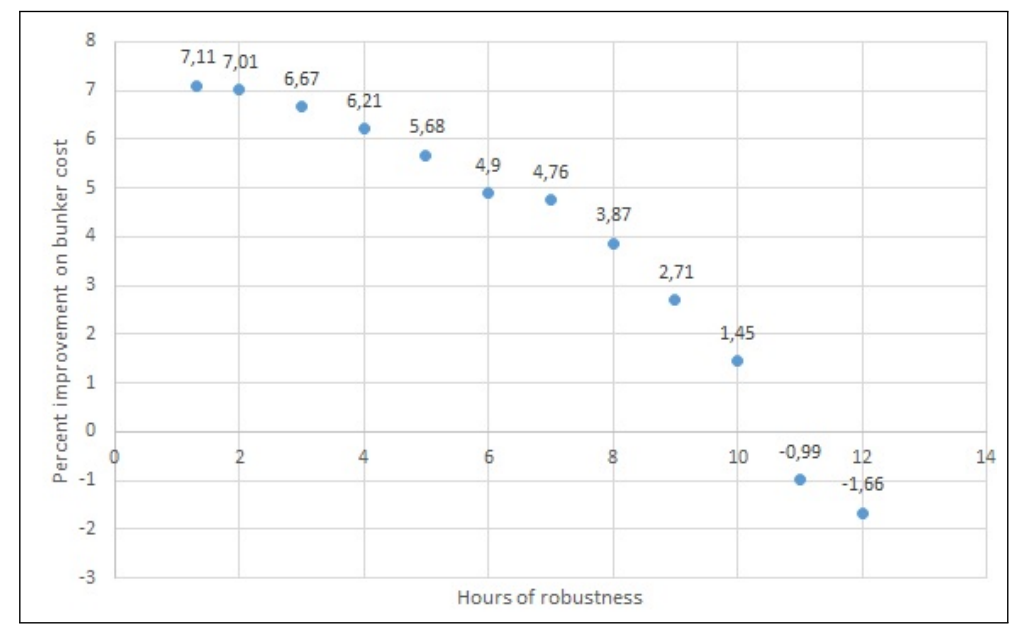

Figure 4: The Pareto optimal frontier of the Single-service 1 instance using the $\epsilon$-constraint method. Each step was solved with a time limit of 600 seconds. The savings in bunker costs (measured in percent improvement) are depicted as function of the robustness (measured in hours).

in each direction during a week. However, it can be seen from Table 4 by comparing Europe-Asia services with Latin America services that the instances with a high utilization of the Panama rail do not have a significantly worse optimality gap.

\subsection{Robustness of schedule}

The need for robustness may depend on different conditions such as winter or summer schedule, also there may be a different need for robustness at the individual services or ports. In order to support decision makers in designing a robust schedule, we generate the full $\epsilon$-frontier for the two instances Single-service 1 and Europe-Asia presented in Figure 4 and 5. For computational reasons we impose a time limit on the solution time, and hence the $\epsilon$-frontier is not exact, but it still gives valuable information. Since the robustness cannot be worse than zero, the starting point for the $\epsilon$-constrained method is $\mu \geq 0$. To ensure that the solutions are efficient, we then maximized $\mu$ for the optimal solution value found. For the instance Single-service 1 presented in Figure 4 this results in an efficient solution $\mu=1$.1. A solution with $\mu=0$ having the same objective value exists, although this solution is not efficient.

For our experiments the $\epsilon$-efficient front is found with steps of one hour, which corresponds to the general unit of time by which the port calls are scheduled. Clearly, increasing the robustness requirement will reduce the solution space and thus increasing the robustness requirement will reduce the bunker saving possibilities. However, the frontiers in Figures 4 and 5 show that increasing the robustness with up to five hours can be achieved while still getting significant fuel savings.

\section{Discussion and conclusion}

We have demonstrated that it is possible to reduce the bunker consumption by 3 - 11 percent by rescheduling the port calls and hence optimize the speed. This is an important step in reaching the IMO goal of a $50 \%$ reduction by 2050 . We also demonstrated in Section 4.8 that significant bunker reduction can be achieved while still ensuring some robustness of the schedule.

Many relevant models have previously been developed for different variants of the speed optimization problem, however, to make the models applicable in practice it is necessary to address a number of business constraints. The model extensions studied in this paper include port work shifts, emission control areas, canal crossings, and piracy areas. The model has been tested on instances representing concrete real-life cases both in terms of size and data. The model extensions are also useful when negotiating vessel sharing agreements in order to optimize the speeds on shared legs to satisfy the connections of both companies while insuring low bunker consumption. VSA agreements can be handled in the speed optimization either by locking some of the times on the service or by sharing the savings proportionally. 


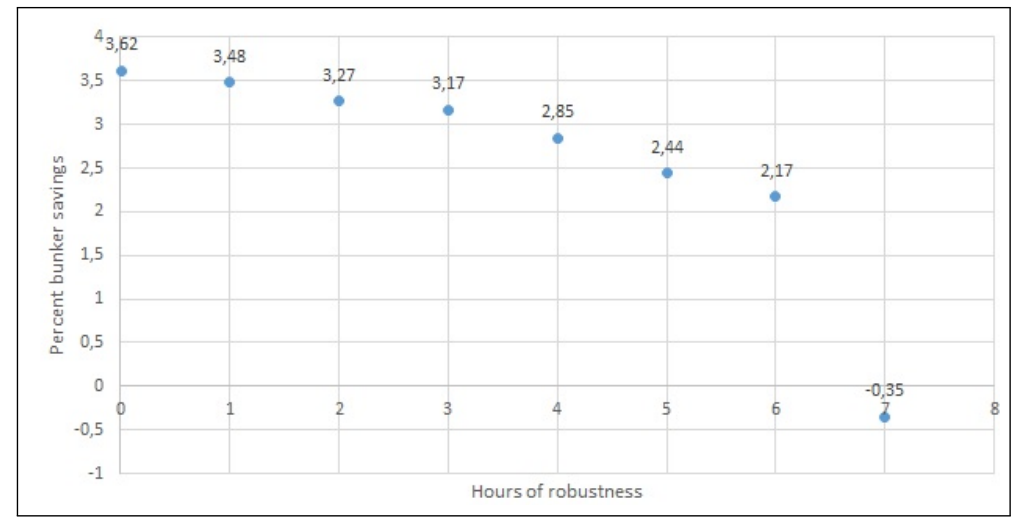

Figure 5: The Pareto optimal frontier of the Europe-Asia services instance using the $\epsilon$-constraint method. Each step was solved with a time limit of 3600 seconds.

It has been demonstrated that, despite the many operational constraints, significant savings can be achieved in real-life cases. Due to the rigorous modeling of all constraints, the found solutions are easier to implement and the promised bunker reduction are close to reality.

The detailed computational study of each operational constraint has furthermore shown how much each constraint affects the fuel consumption, and hence which constraints it would be good to relax in the future to achieve better fuel economy. In particular, we have seen that VSA agreements pose the main obstacle in optimizing the speed. This important observation should be taken into account when companies invest in new vessels in the future. The current trend is to build still bigger vessels, but bigger vessels need VSA agreements to ensure proper utilization of the capacity.

According to the computational experiments, around $4 \%$ reduction can easily be achieved for a complete network without investing in additional vessels, and without significant delays of the cargo. However, to reach the IMO goal of $50 \% \mathrm{CO} 2$ reduction in 2050, it may be necessary with additional speed reduction, and hence extending the fleet. The developed model makes it possible to experiment with additional vessels on each service, and hence further speed reductions. Such a study makes it possible to estimate how many additional vessels are needed to reach a given emission target, and to study how the resulting transit times are affected.

Speed optimization models can also be adapted by authorities to evaluate the effect of emission policies on the actual emission of the operation assuming that the liner shipping company will try to minimize their bunker cost while satisfying the regulations. When modeling emission policies it is again important that the model reflects the operations correctly in order to get the proper picture.

In order to reach the IMO goal of $50 \% \mathrm{CO} 2$ reduction, it may be necessary to differentiate cargo with different time demands. A designated high-speed network can serve perishable goods, refrigerated containers, and high-value freight, while a slow-steaming network can serve the remaining bulk of cargo. Further research is needed to study the design and operation of such a layered network.

It is clear from the results that optimizing more than one service affects the running time. Thus another future research direction could be to develop a heuristic method optimizing each service individually in an iterative fashion.

\section{References}

[1] R. Agarwal and O. Ergun. Ship scheduling and network design for cargo routing in liner shipping. Transportation Science, 42(2):175-196, 2008.

[2] P. M. Alderton. Sea Transport: Operation and Economics (Reed's Professional). Thomas Reed Publications, UK, 2004. ISBN: 9780713669442 .

[3] J. F. Álvarez. Joint routing and deployment of a fleet of container vessels. Maritime Economics and Logistics, 11(2):186$208,2009$. 
[4] B. Brouer, C. Karsten, and D. Pisinger. Optimization in liner shipping. 4OR, 15(1), 2017.

[5] B. D. Brouer, J. F. Alvarez, C. E. M. Plum, D. Pisinger, and M. M. Sigurd. A base integer programming model and benchmark suite for liner-shipping network design. Transportation Science, 48(2):281-312, 2014.

[6] D. G. Carmichael. Computation of pareto optima in structural design. International Journal for Numerical Methods in Engineering, 15(6):925-929, 1980.

[7] M. Christiansen, K. Fagerholt, B. Nygreen, and D. Ronen. Chapter 4 maritime transportation. Handbooks in Operations Research and Management Science, 14(C):189-284, 2007.

[8] M. Christiansen, K. Fagerholt, B. Nygreen, and D. Ronen. Ship routing and scheduling in the new millennium. European Journal of Operational Research, 228(3):467-483, 2013.

[9] M. Christiansen, K. Fagerholt, and D. Ronen. Ship routing and scheduling: Status and perspectives. Transportation Science, 38(1):1-18, 2004.

[10] P. Dithmer, L. Reinhardt, and C. A. Kontovas. The liner shipping routing and scheduling problem under environmental considerations: The case of emissions control areas. Lecture Notes in Computer Science (including subseries Lecture Notes in Artificial Intelligence and Lecture Notes in Bioinformatics), 10572 LNCS:336-350, 2017.

[11] M. Ehrgott and X. Gandibleux. A survey and annotated bibliography of multiobjective combinatorial optimization. $O R$ Spektrum, 22(4):425-460, 2000.

[12] K. Fagerholt, N. T. Gausel, J. G. Rakke, and H. N. Psaraftis. Maritime routing and speed optimization with emission control areas. Transportation Research Part C: Emerging Technologies, 52:57-73, 2015.

[13] K. Fagerholt and H. N. Psaraftis. On two speed optimization problems for ships that sail in and out of emission control areas. Transportation Research Part D: Transport and Environment, 39:56-64, 2015.

[14] E. Gkanatsas, E. U. R. F. der Economische Wetenschappen, M. in Maritime Economics, and Logistics. Designing Robust Shipping Schedules. Erasmus University, 2005.

[15] R. L. Graham. An efficient algorithm for determining the convex hull of a finite planar set. Information Processing Letters, 1(4):132-133, 1972.

[16] S. Guericke and K. Tierney. Liner shipping cargo allocation with service levels and speed optimization. Transportation Research Part E: Logistics and Transportation Review, 84:40-60, 2015.

[17] Y. V. Haimes, L. S. Lasdon, and D. A. Wismer. On a bicriterion formation of the problems of integrated system identification and system optimization. IEEE Transactions on Systems, Man and Cybernetics, SMC-1(3):296-297, 1 1971.

[18] C. Iris, D. Pacino, S. Ropke, and A. Larsen. Integrated berth allocation and quay crane assignment problem: Set partitioning models and computational results. Transportation Research Part E: Logistics and Transportation Review, $81: 75-97,2015$.

[19] C. V. Karsten, B. D. Brouer, G. Desaulniers, and D. Pisinger. Time constrained liner shipping network design. Transportation Research Part E: Logistics and Transportation Review, 105:152-162, 2017.

[20] C. V. Karsten, D. Pisinger, S. Ropke, and B. D. Brouer. The time constrained multi-commodity network flow problem and its application to liner shipping network design. Transportation Research Part E: Logistics and Transportation Review, 76:122-138, 2015.

[21] S. Ozcan, D. T. Eliiyi, and L. B. Reinhardt. Cargo allocation and vessel scheduling on liner shipping with synchronization of transshipments. Applied Mathematical Modelling, 77:235-252, 2020.

[22] W. Passchyn, D. Briskorn, and F. C. R. Spieksma. Mathematical programming models for lock scheduling with an emission objective. European Journal of Operational Research, 248(3):802-814, 2016.

[23] H. N. Psaraftis. Green Transportation Logistics: The Quest for Win-Win Solutions. 2015.

[24] H. N. Psaraftis and C. A. Kontovas. Speed models for energy-efficient maritime transportation: A taxonomy and survey. Transportation Research Part C: Emerging Technologies, 26:331-351, 2013.

[25] K. Rana and R. G. Vickson. Routing container ships using lagrangean relaxation and decomposition. Transportation Science, 25(3):201-214, 1991.

[26] L. Reinhardt, B. Kallehauge, A. Nielsen, and A. Olsen. Network Design Models for Container Shipping. Technical University of Denmark, Centre for Traffic and Transport, 2007. ISBN: 978-87-91137-22-5. 
[27] L. B. Reinhardt and D. Pisinger. A branch and cut algorithm for the container shipping network design problem. Flexible Services and Manufacturing Journal, 24(3):349-374, 2012.

[28] L. B. Reinhardt, C. E. M. Plum, D. Pisinger, M. M. Sigurd, and G. T. P. Vial. The liner shipping berth scheduling problem with transit times. Transportation Research Part E: Logistics and Transportation Review, 86:116-128, 2016.

[29] M. Stopford. Maritime Economics. Routledge, 2009.

[30] C. Wang and C. Xu. Sailing speed optimization in voyage chartering ship considering different carbon emissions taxation. Computers and Industrial Engineering, 89:108-115, 2015.

[31] S. Wang. Fundamental properties and pseudo-polynomial-time algorithm for network containership sailing speed optimization. European Journal of Operational Research, 250(1):46-55, 2016.

[32] S. Wang and Q. Meng. Liner ship route schedule design with sea contingency time and port time uncertainty. Transportation Research Part B: Methodological, 46(5):615-633, 2012.

[33] S. Wang and Q. Meng. Robust schedule design for liner shipping services. Transportation Research Part E: Logistics and Transportation Review, 48(6):1093-1106, 2012.

[34] S. Wang and Q. Meng. Sailing speed optimization for container ships in a liner shipping network. Transportation Research Part E: Logistics and Transportation Review, 48(3):701-714, 2012.

[35] S. Wang, Q. Meng, and C.-Y. Lee. Liner container assignment model with transit-time-sensitive container shipment demand and its applications. Transportation Research Part B: Methodological, 90:135-155, 2016.

[36] O. Weide, D. Ryan, and M. Ehrgott. An iterative approach to robust and integrated aircraft routing and crew scheduling. Computers and Operations Research, 37(5):833-844, 2010.

[37] D. White. Epsilon efficiency. Journal of Optimization Theory and Applications, 49(2):319-337, 1986. 